\title{
Genomic diversity of Escherichia coli from healthy children in rural Gambia
}

\author{
Ebenezer Foster-Nyarko ${ }^{1,2}$, Nabil-Fareed Alikhan ${ }^{1}$, Usman N Ikumapayi ${ }^{2}$, Golam Sarwar ${ }^{2}$, M Jahangir Hossain ${ }^{2}$, \\ Catherine Okoi ${ }^{2}$, Peggy-Estelle Maguiagueu Tientcheu ${ }^{2}$, Marianne Defernez ${ }^{1}$, Justin O'Grady ${ }^{1}$, Martin Antonio ${ }^{2,3}$, \\ Mark J Pallen ${ }^{\text {Corresp. 1, } 4}$ \\ 1 Quadram Institute Bioscience, Norwich Research Park, Norfolk, United Kingdom \\ 2 Medical Research Council Unit The Gambia at the London School of Hygiene and Tropical Medicine, Fajara, The Gambia \\ 3 Microbiology and Infection Unit, Warwick Medical School, University of Warwick, Coventry, United Kingdom \\ ${ }^{4}$ School of Veterinary Medicine, University of Surrey, Surrey, United Kingdom \\ Corresponding Author: Mark J Pallen \\ Email address: m.pallen@warwick.ac.uk
}

Little is known about the genomic diversity of Escherichia coli in healthy children from subSaharan Africa, even though this is pertinent to understanding bacterial evolution and ecology and their role in infection. We isolated and whole-genome sequenced up to five colonies of faecal E. coli from 66 asymptomatic children aged three-to-five years in rural Gambia ( $n=88$ isolates from 21 positive stools). We identified 56 genotypes, with an average of 2.7 genotypes per host. These were spread over 37 seven-allele sequence types and the E. coli phylogroups A, B1, B2, C, D, E, F and Escherichia cryptic clade I. Immigration events accounted for three-quarters of the diversity within our study population, while one-quarter of variants appeared to have arisen from within-host evolution. Several isolates encode putative virulence factors commonly found in Enteropathogenic and Enteroaggregative E. coli, and 53\% of the isolates encode resistance to three or more classes of antimicrobials. Thus, resident $E$. coli in these children may constitute reservoirs of virulence- and resistance-associated genes. Moreover, several study strains were closely related to isolates that caused disease in humans or originated from livestock. Our results suggest that within-host evolution plays a minor role in the generation of diversity compared to independent immigration and the establishment of strains among our study population. Also, this study adds significantly to the number of commensal E. coli genomes, a group that has been traditionally underrepresented in the sequencing of this species. 


\section{Genomic diversity of Escherichia coli isolates from healthy children in rural Gambia}

2 Ebenezer Foster-Nyarko ${ }^{1,2}$, Nabil-Fareed Alikhan ${ }^{1}$, Usman N Ikumapayi $^{2}$, Sarwar Golam²,

3 Catherine Okoi ${ }^{2}$, Peggy-Estelle Maguiagueu Tientcheu ${ }^{2}$, Marianne Defernez ${ }^{1}$, Justin O'Grady ${ }^{1}$,

4 Martin Antonio ${ }^{2,3}$, Mark J. Pallen ${ }^{1,4 \#}$

$7{ }^{1}$ Quadram Institute Bioscience, Norwich Research Park, Norwich, Norfolk, United Kingdom

$8{ }^{2}$ Medical Research Council Unit The Gambia at the London School of Hygiene and Tropical

9 Medicine, Atlantic Boulevard Road, Fajara, the Gambia

$10{ }^{3}$ Warwick Medical School, University of Warwick, Coventry, United Kingdom

$11{ }^{4}$ School of Veterinary Medicine, University of Surrey, Guildford, Surrey, United Kingdom

$14{ }^{\#}$ Corresponding author: Professor Mark J. Pallen, Quadram Institute Bioscience, Norwich

15 Research Park, Norwich, Norfolk, United Kingdom

16 Email: Mark.Pallen@quadram.ac.uk 


\section{Abstract}

19 Little is known about the genomic diversity of Escherichia coli in healthy children from sub-

20 Saharan Africa, even though this is pertinent to understanding bacterial evolution and ecology

21 and their role in infection. We isolated and whole-genome sequenced up to five colonies

22 of faecal $E$. coli from 66 asymptomatic children aged three-to-five years in rural Gambia (n=88

23 isolates from 21 positive stools). We identified 56 genotypes, with an average of 2.7 genotypes

24 per host. These were spread over 37 seven-allele sequence types and the E. coli phylogroups A,

25 B1, B2, C, D, E, F and Escherichia cryptic clade I. Immigration events accounted for three-

26 quarters of the diversity within our study population, while one-quarter of variants appeared to

27 have arisen from within-host evolution. Several isolates encode putative virulence factors

28 commonly found in Enteropathogenic and Enteroaggregative E. coli, and 53\% of the isolates

29 encode resistance to three or more classes of antimicrobials. Thus, resident $E$. coli in these

30 children may constitute reservoirs of virulence- and resistance-associated genes. Moreover,

31 several study strains were closely related to isolates that caused disease in humans or originated

32 from livestock. Our results suggest that within-host evolution plays a minor role in the

33 generation of diversity compared to independent immigration and the establishment of strains

34 among our study population. Also, this study adds significantly to the number of commensal $E$.

35 coli genomes, a group that has been traditionally underrepresented in the sequencing of this

36 species.

37

38 Keywords: Escherichia coli, genomic diversity, within-host evolution. 


\section{Introduction}

42 Ease of culture and genetic tractability account for the unparalleled status of Escherichia coli as

43 "the biological rock star", driving advances in biotechnology (Blount 2015), while also providing

44 critical insights into biology and evolution (Good et al. 2017). However, E. coli is also a

45 widespread commensal, as well as a versatile pathogen, linked to diarrhoea (particularly in the

46 under-fives), urinary tract infection, neonatal sepsis, bacteraemia and multi-drug resistant

47 infection in hospitals (Camins et al. 2011; Rodríguez-Baño et al. 2010; Russo \& Johnson 2003).

48 Yet, most of what we know about E. coli stems from the investigation of laboratory strains,

49 which fail to capture the ecology and evolution of this key organism "in the wild" (Hobman et al.

50 2007). What is more, most studies of non-lab strains have focused on pathogenic strains or have

51 been hampered by low-resolution PCR methods, so we have relatively few genomic sequences

52 from commensal isolates, particularly from low- to middle-income countries (Ahmed et al. 2014;

53 Ferjani et al. 2017; Moremi et al. 2017; Oshima et al. 2008; Rasko et al. 2008; Stoesser et al.

54 2015; Touchon et al. 2009).

55 We have a broad understanding of the population structure of $E$. coli, with eight significant

56 phylogroups loosely linked to ecological niche and pathogenic potential (B2, D and F linked to

57 extraintestinal infection; A and B1 linked to severe intestinal infections such as haemolytic-

58 uraemic syndrome) (Alm et al. 2011; Escobar-Paramo et al. 2004; Mellata 2013; Walk et al.

59 2009). All phylogroups can colonise the human gut, but it remains unclear how far commensals

60 and pathogenic strains compete or collaborate - or engage in horizontal gene transfer — within

61 this important niche (Laxminarayan et al. 2013; Stoppe et al. 2017).

62 Although clinical microbiology typically relies on single-colony picks (which has the

63 potential to underestimate species diversity and transmission events), within-host diversity of $E$. 
64 coli in the gut is crucial to our understanding of inter-strain competition and co-operation and

65 also for accurate diagnosis and epidemiological analyses. Pioneering efforts using serotyping and

66 molecular typing have shown that normal individuals typically harbour more than one strain of

67 E. coli (Chen et al. 2013; Dixit et al. 2018; Richter et al. 2018), with one individual carrying 24

68 distinct clones (Chen et al. 2013; Schlager et al. 2002; Shooter et al. 1977). More recently,

69 whole-genome sequencing has illuminated molecular epidemiological investigations (Stoesser et

70 al. 2015), for example, studies of the transmission of extended-spectrum beta-lactamase-

71 encoding E. coli, multidrug-resistant Acinetobacter baumannii, and the genomic surveillance of

72 multidrug-resistant E. coli carriage. Whole-genome data has also been applied to studies of $E$.

73 coli adaptation during and after infection (McNally et al. 2013; Nielsen et al. 2016), as well as

74 the intra-clonal diversity in healthy hosts (Stegger et al. 2020).

75 There are two plausible sources of within-host genomic diversity. Although a predominant

76 strain usually colonises the host for extended periods (Hartl \& Dykhuizen 1984), successful

77 immigration events mean that incoming strains can replace the dominant strain or co-exist

78 alongside it as minority populations (Bettelheim et al. 1972). Strains originating from serial

79 immigration events are likely to differ by hundreds or thousands of single-nucleotide

80 polymorphisms (SNPs). Alternatively, within-host evolution can generate clouds of intra-clonal

81 diversity, where genotypes differ by just a handful of SNPs (Dixit et al. 2018).

82 Most relevant studies have been limited to Western countries, except for a recent report from

83 Tanzania (Richter et al. 2018), so little is known about the genomic diversity of E. coli in sub-

84 Saharan Africa. The Global Enteric Multicenter Study (GEMS) (Kotloff et al. 2013; Liu et al.

85 2016) has documented a high burden of diarrhoea attributable to E. coli (including Shigella)

86 among children from the Gambia, probably as a result of increased exposure to this organism 
87 through poor hygiene and frequent contact with animals and the environment. GEMS was a

88 prospective case-control study which investigated the aetiology of moderate-to-severe diarrhoea

89 in children aged less than five years residing in sub-Saharan Africa and South Asia. In the

90 Gambia, children with moderate-to-severe diarrhoea seeking care at the Basse Health centre in

91 the Upper River Division of the country were recruited, with one to three matched control

92 children randomly selected from the community along with each case. In also facilitating access

93 to stool samples from healthy Gambian children, the GEMS study has given us a unique

94 opportunity to study within-host genomic diversity of commensal E. coli in this setting.

\section{Methods}

\section{Study population}

98 We initially selected 76 faecal samples from three- to five-four-old (36-59 months) asymptomatic Gambian children, who had been recruited into the GEMS study (Kotloff et al.

100 2013) as healthy controls from December 1, 2007, to March 3, 2011. Samples had been collected

101 according to a previously described sampling protocol (Kotloff et al. 2012) and the results of the

102 original study are publicly available at ClinEpiDB.org. Ten of the original 76 samples were

103 depleted and were therefore unavailable for processing in this study. Of the remaining 66 stools,

10462 had previously tested positive for E. coli. GEMS isolated three E. coli colonies per stool

105 sample but pooled these into a single tube for frozen storage. Thus, we needed to re-culture the

106 stools with multiple colony picks, as the original isolate collection was unsuitable for the

107 investigation of within-host diversity. Archived stool samples were retrieved from $-80^{\circ} \mathrm{C}$ storage

108 and allowed to thaw on ice. A 100-200 mg aliquot from each sample was transferred aseptically

109 into $1.8 \mathrm{ml}$ Nunc tubes for microbiological processing below (Figure 1). 


\section{Bacterial growth and isolation}

$1121 \mathrm{ml}$ of physiological saline $(0.85 \%)$ was added to each sample tube and vigorously vortexed at

$1134200 \mathrm{rpm}$ for at least 2 minutes. Next, the homogenised sample suspensions were taken through

114 four ten-fold dilution series. A100 $\mu 1$ aliquot from each dilution was then spread evenly on a

115 plate of tryptone-bile-X-glucuronide differential and selective agar. The inoculated plates were

116 incubated overnight at $37^{\circ} \mathrm{C}$ under aerobic conditions. Colony counts were performed on the

117 overnight cultures for each serial dilution for translucent colonies with entire margins and blue-

118 green pigmentation indicative of E. coli. Up to five representative colonies were selected from

119 each sample and sub-cultured on MacConkey agar overnight at $37^{\circ} \mathrm{C}$ before storing in $20 \%$

120 glycerol broth at $-80^{\circ} \mathrm{C}$. Individual isolates were assigned a designation comprised of the subject

121 ID followed by the colony number ("1-5").

122

123 Genomic DNA extraction and genome sequencing

124 Broth cultures were prepared from pure, fresh cultures of each colony-pick in $1 \mathrm{ml}$ Luria-Bertani

125 broth and incubated overnight to attain between $10^{9}-10^{10} \mathrm{cfu}$ per ml. Genomic DNA was then

126 extracted from the overnight broth cultures using the lysate method described in (Foster-Nyarko

127 et al. 2020). The eluted DNA was quantified by the Qubit high sensitivity DNA assay kit

128 (Invitrogen, MA, USA) and sequenced on the Illumina NextSeq 500 instrument (Illumina, San

129 Diego, CA), using a modified Nextera XT DNA protocol for the library preparation as described

130 previously (Foster-Nyarko et al. 2020). The pooled library was loaded on a mid-output flow cell

131 (NSQ 500 Mid Output KT v2 300 cycles; Illumina Catalogue No. FC-404-2003) at a final 
132 concentration of $1.8 \mathrm{pM}$, following the Illumina recommended denaturation and loading

133 parameters - including a 1\% PhiX spike (PhiX Control v3; Illumina Catalogue FC-110-3001).

134 Following Dixit et al. (Dixit et al. 2018), we sequenced a random selection of ten isolates

135 twice, using DNA obtained from independent cultures, to help in the determination of clones and

136 the analysis of within-host variants (Supplementary File 1). Bioinformatic analyses of the

137 genome sequences were carried out on the Cloud Infrastructure for Microbial Bioinformatics

138 (CLIMB) platform (Connor et al. 2016).

140 Genome assembly and phylogenetic analysis

141 The paired $150 \mathrm{bp}$ reads were quality checked and concatenated, then quality checked using the

142 FastQC tool v0.11.7 (Wingett \& Andrews 2018) and assembled using SPAdes genome assembler

143 v3.12.0 (Bankevich et al. 2012), under default parameters. The quality of the assemblies was

144 assessed using QUAST v5.0.0, de6973bb (Gurevich et al. 2013). We used Snippy v4.3.2

145 (https://github.com/tseemann/snippy) — a rapid command line tool that finds SNPs (substitutions

146 and insertions/deletions) between a haploid reference genome and input sequence reads and

147 generates a core SNP alignment which can be used to reconstruct a high-resolution phylogeny-

148 to generate a core-genome alignment based on core SNPs under default parameters. The

149 complete genome sequence of commensal E. coli str. K12 substr. MG1655 as a reference strain

150 (NCBI accession: NC_000913.3). From the core-genome alignment, we then reconstructed a

151 maximum-likelihood phylogeny with 1000 bootstrap replicates using RAxML v8.2.4

152 (Stamatakis 2006), based on a general time-reversible nucleotide substitution model. The

153 phylogenetic tree was rooted using the genomic sequence of E. fergusonii as an outgroup (NCBI

154 accession: GCA_000026225.1). The phylogenetic tree was visualised in FigTree v1.4.3 
155 (https://github.com/rambaut/figtree/) and annotated in RStudio v3.5.1 and Adobe Illustrator v

156 23.0.3 (Adobe Inc., San Jose, California). As recombination is known to be widespread in E. coli

157 and can blur phylogenetic signals (Wirth et al. 2006), we detected and masked any recombinant

158 regions of the core-genome alignment using Gubbins (Genealogies Unbiased By recomBinations

159 In Nucleotide Sequences) (Croucher et al. 2015) before the phylogenetic reconstruction. For

160 visualisation, a single colony was chosen to represent replicate colonies of the same strain (ST)

161 with identical virulence, plasmid and antimicrobial resistance profiles and a de-replicated

162 phylogenetic tree reconstructed using the representative isolates. We computed pairwise single

163 nucleotide polymorphism (SNP) distances between genomes from the core-genome alignment

164 using snp-dists v0.6 (https://github.com/tseemann/snp-dists).

165

166 Multi-locus sequence typing, Clermont typing

167 The merged reads were uploaded to EnteroBase (Zhou et al. 2020), where de novo assembly and 168 genome annotation were carried out, and in-silico multi-locus sequence types (MLST) assigned

169 based on the Achtman scheme, allocating new sequence types (ST) if necessary. EnteroBase

170 assigns phylogroups using ClermontTyper and EzClermont (Clermont et al. 2013; Clermont et

171 al. 2015) and unique core-genome MLST types (cgMLST) based on 2, 513 core loci in E. coli.

172 Publicly available E. coli sequences in EnteroBase

173 (http://enterobase.warwick.ac.uk/species/index/ecoli) (Zhou et al. 2020) were included for

174 comparative analysis, including 23 previously sequenced isolates obtained from diarrhoeal cases

175 recruited in the GEMS study in the Gambia (Supplementary File 2). The isolates can be

176 searched in EnteroBase using the 'Search Strains' parameter and under 'Strain Metadata', 
177 selecting the 'Name' option and entering the study sample name (column 1 of Supplementary

178 File 2) in the 'Value' box.

179

180 Determination of immigration events and within-host variants

181 For the whole genome sequences of the strains sequenced twice, we used SPAdes v3.13.2

182 (Bankevich et al. 2012) to assemble each set of reads and map the raw sequences from one

183 sequencing run to the assembly of the other run and vice versa, as described previously (Dixit et

184 al. 2018). Briefly, mapping was done using the BWA-MEM algorithm v0.7.17-r1188 under

185 default parameters to generate a SAM alignment. This was then converted to BAM files using

186 Samtools view v1.9 (Li et al. 2009), sorted and indexed. Next, variants were called and written to

187 a VCF file using Samtools mpileup and the "view" module of BCFtools (which is part of the

188 Samtools v1.9 package) and visualised in Tablet v1.19.09.13 (Milne et al. 2013). The number of

189 SNPs, and their positions were determined and compared between the two steps, counting only

190 those SNPs that were detected in both sets of reads as accurate.

191 In line with (Dixit et al. 2018), isolates belonging to different STs recovered from the same

192 host were considered to be separate strains derived from independent exposures and immigration

193 events. As described in (Dixit et al. 2018), we determined the number of SNP differences that

194 existed between assemblies of the same isolate that were sequenced on two separate occasions,

195 to determine if multiple isolates of the same ST from a single host were distinct variants (clones).

196 If the SNP difference between two isolates belonging to the same ST recovered from the same

197 host was less than the SNP difference between the sequences of the same isolate sequenced on

198 two separate occasions, then the two isolates were taken to represent replicate copies of the same

199 clone. Otherwise, they were considered as within-host variants (separate, distinct clones of the 
200 same strain) - provided the SNP differences between such distinct clones were no more than

201 eleven SNPs. This cut-off was chosen based on an estimated mutation rate of 1.1 SNP per

202 genome per year (Reeves et al. 2011), assuming equal rates of mutation in both genomes being

203 compared. Based on these data, we inferred replicate clones with SNP differences of greater than

20411 SNPs to represent a divergence of more than five years. Thus, it seems implausible that such

205 replicate clones would have emerged from within-host evolution, considering the age of the 206 study participants $(<5$ years old $)$.

207 We produced a contingency table to summarise the distribution of variants derived from 208 migration events and within-host evolution and visualised this using a clustered bar graph. We

209 then performed Fisher's exact test to investigate the association between phylogroup and the

210 distribution of variants (migration versus within-host evolution). Our calculations were based on

211 the assumption of independence among the observed phylogroups - that is, the finding of one

212 phylogroup does not preclude or predict the co-occurrence of another.

213

\section{Accessory gene content}

215 We used ABRicate v0.9.8 (https://github.com/tseemann/abricate) to predict virulence factors,

216 acquired antimicrobial resistance (AMR) genes and plasmid replicons by scanning the contigs

217 against the VFDB, ResFinder and PlasmidFinder databases respectively, using an identity

218 threshold of $\geq 90 \%$ and a coverage of $\geq 70 \%$. Virulence factors and AMR genes were plotted

219 next to the phylogenetic tree using the ggtree, ggplot2 and phangorn packages in RStudio v3.5.1.

220 We calculated co-occurrence of AMR genes among study isolates by transforming the binary

221 AMR gene content matrix and visualising this as a heat map using the pheatmap package $\mathrm{v}$

2221.0 .12 (https://CRAN.R-project.org/package=pheatmap) in RStudio v3.5.1. We computed 
223 Fisher's exact tests between the detected virulence factors and the observed phylogroups in

224 RStudio v3.5.1.

225

226 Population structure and comparison of commensal and pathogenic strains

227 We assessed the population structure using the hierarchical clustering algorithm in EnteroBase.

228 Briefly, the isolates were assigned stable population clusters at eleven levels (from $\mathrm{HC} 0$ to $\mathrm{HC}$

229 2350) based on pairwise cgMLST allelic differences. Hierarchical clustering at 1100 alleles

230 differences (HC1100) resolves populations into cgST (core-genome MLST type) complexes, the

231 equivalent of clonal complexes achieved with the legacy MLST clustering approaches (Zhou et

232 al. 2020). We reconstructed neighbour-joining phylogenetic trees using NINJA (Wheeler 2009),

233 based on clustering at $\mathrm{HC} 1100$ to display the population sub-clusters at this level as an indicator

234 of the genomic diversity within our study population and to infer the evolutionary relationship

235 among our strains and others in the public domain.

236 Next, we interrogated the HC1100 clusters that encompassed our study isolates and Gambian

237 pathogenic isolates recovered from diarrhoeal cases and commensal E. coli strains recovered

238 from the GEMS study. For the clusters that encompassed commensal and pathogenic strains

239 belonging to the same ST (HC1100_200 cluster, comprising pathogenic isolates from GEMS

240 cases 100415, 102106 and 102098 and the resident ST38 strain recovered from our study subject

241 18), we reconstructed both neighbour-joining and SNP phylogenetic trees to display the genetic

242 relationships among these strains. We visualised the accessory genomes for the overlapping STs

243 mentioned above to determine genes associated with phages, virulence factors and AMR. The

244 resulting phylogenetic trees were annotated in Adobe Illustrator v 23.0.3 (Adobe Inc., San Jose,

245 California). 


\section{Ethical statement}

248 The parent study was approved by the joint Medical Research Council Unit The Gambia-

249 Gambian Government ethical review board (SCC 1331). Written informed consents were

250 obtained from all the study participants as previously reported in (Kotloff et al. 2013). The joint

251 Medical Research Council Unit The Gambia-Gambian Government ethical review board gave 252 approval for the use of the stool samples analysed in this study.

253

254 Results

255 Population structure

256 The study population included 27 females and 39 males (Supplementary File 3). All but one

257 reported the presence of a domestic animal within the household. Twenty-one samples proved 258 positive for the growth of E. coli, yielding 88 isolates (Supplementary File 4). We detected 37

259 seven-allele sequence types (STs) among the isolates, with a fairly even distribution (Figure 2).

260 Five STs were completely novel (ST9274, ST9277, ST9278, ST9279 and ST9281). These study

261 strains were scattered over all the eight main phylogroups of E. coli: A (27\%), B1 (32\%), B2

262 (9\%), D (15\%), C and F (5\% each), E (1\%), and the cryptic Clade I (7\%), although the majority

263 belonged to phylogroups A and B1 (Table 1). Hierarchical clustering of core genomic STs

264 revealed twenty-seven cgST clonal complexes (Supplementary File 4). The raw genomic

265 sequences of the study isolates have been deposited in the NCBI SRA under the BioProject ID

266 PRJNA658685 (accession numbers SAMN15880274 to SAMN15880361).

268 Within-host diversity 
269 Just a single ST colonised nine individuals, six carried two STs, four carried four STs, and two

270 carried six STs. We found 56 distinct genotypes, which equates to an average of 2.7 genotypes

271 per host. Two individuals (H-18 and H-2) shared an identical strain belonging to ST9274 (zero

272 SNP difference) (Supplementary File 5, yellow highlight), suggesting recent transfer from one

273 child to another or recent acquisition from a common source.

274 We observed thirteen within-host variants in ten hosts (intra-clonal diversity) (subjects H-15, 275 H-18, H-22, H-25, H-28, H-34, H36, H37, H-38 and H-39), compared to forty-one immigration

276 events (Tables 1 and 2). Overall, immigration events accounted for the majority (76\%) of

277 variants (Supplementary Figure 1). The proportion of migration versus within-host evolution

278 events did not appear to be affected by phylogroup $(\mathrm{p}=0.42)$. Twenty-two percent of within-host

279 mutations represented synonymous changes, $43 \%$ were non-synonymous mutations, while $31 \%$

280 occurred in non-coding regions, and 4\% represented stop-gained mutations (Supplementary File

281 6). On an average, $\mathrm{Ka} / \mathrm{Ks}$ ratios were greater than 1 , which seems to suggest that these mutations

282 were under positive Darwinian selection - indicating that most of the mutations were likely to

283 have little effect on fitness. However, these remain to be investigated further. Also, the observed

284 non-synonymous mutations were spread across genes with a variety of functions, including

285 metabolism, transmembrane transport, pathogenesis and iron import into the cell. However, the

286 bulk (42\%) occurred in genes involved in metabolism. The average number of SNPs among

287 within-host variants was 5 (range 0-18) (Table 2). However, in two subjects (H36 and H37),

288 pairwise distances between genomes from the same ST (ST59 and ST5148) were as large as 14

289 and 18 SNPs respectively (Supplementary File 5, grey highlight).

290

291 Accessory gene content and relationships with other strains 
292 A quarter of our isolates were most closely related to commensal strains from humans, with

293 smaller numbers most closely related to human pathogenic strains or strains from livestock,

294 poultry or the environment (Supplementary File 7). One isolate was most closely related to a

295 canine isolate from the UK. Three STs (ST38, ST10 and ST58) were shared by our study isolates

296 and diarrhoeal isolate from the GEMS study (Supplementary Figure 2), with just eight alleles

297 separating our commensal ST38 strain from a diarrhoeal isolate from the GEMS study (Figure

298 3). For ST10 and ST58, hierarchical clustering placed the commensal strains from this study into

299 separate clusters from the pathogenic isolates from diarrhoeal cases, indicating that they were

300 genetically distinct to each other. Yet, the closest relative of our study ST58 strain was an

301 extraintestinal strain isolated from the blood of a 69 -year-old male (87 alleles differences, Figure

302 4). Also, the resident ST10 isolates recovered from this study (H-26_2, H-34_2, and H-32_5) had

303 their closest neighbours in isolates from livestock (83 and 111 alleles each), and an isolate of an

304 unspecified source (18 alleles differences) respectively (Supplementary File 7).

305 We detected 130 genes encoding putative virulence factors across the 88 study isolates

306 (Figure 2; Supplementary File 8). Notable among these were genes associated with pathogenesis

307 in Enteroaggregative E. coli and Salmonella referred to as the Serine Protease Autotransporters

308 of Enterobacteriaceae (SPATEs) (Pokharel et al. 2019), such as sat (13\%), sigA (11\%) and pic

309 (1\%). Besides, eight isolates harboured known markers of Enteropathogenic E. coli (eltAB or

310 estA). Several strains (across all phylogroups) also harboured virulence genes associated with

311 intestinal or extraintestinal disease in humans, including adhesins, invasins, toxins and iron-

312 acquisition genes such as fyuA, several fim and pap genes, iroN, irp 1,2, ibeA and aslA. We did

313 not detect any of the well-known markers of EPEC (eae, bfpA, stx 1, or stx2) (Figure 2,

314 Supplementary File 8). 
315 The prevalence of some virulence factors involved in invasion/evasion, iron uptake,

316 adherence, and secretion systems appeared to be more or less likely to occur in one or a few

317 phylotypes $(\mathrm{p} \leq 0.05)$ as follows (Supplementary File 9). The iron acquisition genes $\operatorname{chuA}, S-Y$ and

318 shuA, $S, T, Y$ were found to be present in all cases for phylogroup $\mathrm{D}(\mathrm{n}=5)$, and absent in

319 virtually all cases for phylogroups $\mathrm{A}(\mathrm{n}=13)$ and $\mathrm{B} 1(\mathrm{n}=16)$. On the other hand, iutA and iucA-D

320 were observed in the two cases from phylogroup B2, and absent from all samples from

321 phylogroup $\mathrm{D}(\mathrm{n}=5)$. The invasion/evasion genes $\mathrm{kps} D, M, T$ and asl $A$ were found to be present

322 in almost all cases for phylogroups D $(n=5), B 2(n=2)$, and Clade $I(n=2)$, and absent in B1

323 ( $\mathrm{n}=16)$. The secretion system gene cluster $\operatorname{esp} B, D, G, K-N, R, W-Y$ was observed in all cases

324 except the two belonging to phylogenetic group B2. The protease gene $\operatorname{sig} A$ was absent from

325 most samples, except two samples from phylotype B2. The adherence gene $f d e C$ was observed in

326 all cases for phylotype $\mathrm{D}(\mathrm{n}=5)$ and most for $\mathrm{B} 1(\mathrm{n}=16)$.

327 More than half of the isolates encoded resistance to three or more clinically relevant classes

328 of antibiotics such as aminoglycosides, penicillins, trimethoprim, sulphonamides and

329 tetracyclines (Figure 5; Supplementary Figure 3). The most common resistance gene network

330 was -aph(6)-Id_1-sul2 (41\% of the isolates), followed by aph(3")-Ib_5-sul2 (27\%) and bla-TEM-

331 aph(3")-Ib_5(24\%). Most isolates (67\%) harboured two or more plasmid types (Figure 6). Of

332 the 24 plasmid types detected, IncFIB was the most common (41\%), followed by col156 (19\%)

333 and IncI_1-Alpha (15\%). Nearly three-quarters of the multi-drug resistant isolates carried IncFIB

334 (AP001918) plasmids ( $\sim 50 \mathrm{~kb})$, suggesting that these large plasmids disseminate resistance genes

335 within our study population.

336

337 Discussion 
338 This study provides an overview of the within-host genomic diversity of E. coli in healthy

339 children from a rural setting in the Gambia, West Africa. Surprisingly, we were able to recover

340 E. coli from only $34 \%$ of stools which had previously tested positive for E. coli in the original

341 study. This low rate of recovery may reflect some hard-to-identify effect of long-term storage

342 (nine to thirteen years) or the way the samples were handled, even though they were kept frozen

343 and thawed only just before culture.

344 Several studies have shown that sampling a single colony is insufficient to capture $E$. coli

345 strain diversity in stools (Dixit et al. 2018; Richter et al. 2018; Shooter et al. 1977). Lidin-Janson

346 et al. (Lidin-Janson et al. 1978) claim that sampling five colonies provides a $>99 \%$ chance of

347 recovering dominant genotypes from single stool specimens, while Schlager et al. (Schlager et

348 al. 2002) calculate that sampling twenty-eight colonies provides a $>90 \%$ chance of recovering

349 minor genotypes. Our results confirm the importance of multiple-colony picks in faecal

350 surveillance studies, as over half (57\%) of our strains would have been missed by picking a

351 single colony.

352 Our strains encompassed all eight major phylotypes of $E$. coli, however, the majority fell into

353 the A and B1 phylogenetic groups, in line with previous reports that these phylogroups dominate

354 in stools from people in low- and middle-income countries (Duriez et al. 2001; Escobar-Páramo

355 et al. 2004). Although not fully understood, there appear to be host-related factors that influence

356 the composition of $E$. coli phylogroups in human hosts. For example, the establishment of strains

357 belonging to phylogroups $\mathrm{E}$ or $\mathrm{F}$ seems to favour subsequent colonisation by other phylotypes,

358 compared to the establishment of phylogroup B2 strains, which tend to limit the heterogeneity

359 within individual hosts (Gordon et al. 2015). Geographical differences have also been reported,

360 with phylogroups A and B1 frequently dominating the stools of people living in developing 
361 countries (Duriez et al. 2001; Escobar-Páramo et al. 2004). Conversely, phylogroup B2 and D

362 strains appear to be pervasive among people living in developed countries (Massot et al. 2016;

363 Skurnik et al. 2008). These locale-specific patterns in the distribution of E. coli phylotypes have

364 been attributed to differences in diet and climate (Duriez et al. 2001; Escobar-Páramo et al.

365 2004).

366 The prevalence of putative virulence genes in most of our isolates highlights the pathogenic

367 potential of commensal intestinal strains - regardless of their phylogroup — should they gain

368 access to the appropriate tissues, for example, the urinary tract. Our results complement previous

369 studies reporting genomic similarities between faecal E. coli isolates and those recovered from

370 urinary tract infection (McNally et al. 2013; Wold et al. 1992).

371 We found that within-host evolution plays a minor role in the generation of diversity in our

372 study population. This might be due to the low prevalence of B2 strains, which are thought to

373 inhibit the establishment of strains from other phylogroups, as discussed above (Gordon et al.

374 2015); or it may indicate that members of phylogroups A and B1 might favour a more

375 heterogeneous composition of $E$. coli phylotypes in stools of healthy individuals. However, this

376 remains to be properly investigated, as we did not find statistical evidence that the distribution of

377 variants (independent migration versus within-host evolution) was influenced by phylogroup.

378 Our findings are similar to that reported, in line with Dixit et al. (Dixit et al. 2018), who reported

379 that $83 \%$ of diversity originates from immigration events, and with epidemiological data

380 suggesting that the recurrent immigration events account for the high faecal diversity of E. coli in

381 the tropics (Tenaillon et al. 2010).

382 The estimated mutation rate for E. coli lineages is around one SNP per genome per year

383 (Reeves et al. 2011), so that two genomes with a most recent common ancestor in the last five 
384 years would be expected to be around ten SNPs apart. However, in two subjects, pairwise

385 distances between genomes from the same ST (ST59 and ST5148) were large enough (14 and 18

386 respectively) to suggest that they might have arisen from independent immigration events, as

387 insufficient time had elapsed in the child's life for such divergence to occur within the host.

388 However, it remains possible that the mutation rate was higher than expected in these lineages,

389 although we found no evidence of damage to DNA repair genes. Co-colonising variants

390 belonging to the same ST tended to share an identical virulence, AMR and plasmid profile,

391 signalling similarities in their accessory gene content.

392 The sources of novel variation that account for within-host diversity include point mutation

393 and small insertions or deletions (indels), indels and the loss or acquisition of mobile genetic

394 elements. Among the variants inferred to have been derived from within-host evolution, we

395 observed dominance of mutations that were predicted to result in changes in protein function, in

396 the form of missense mutations and non-sense mutations (leading to a premature stop codon).

397 Although the mutations appeared to be heterogeneously distributed, a higher number was

398 observed in genes associated with metabolism. These appeared to be under positive selection,

399 although it remains to be seen if these changes confer any effects on fitness. It will be desirable

400 to investigate this in future studies. Due to the cross-sectional nature of our sampling, we were

401 unable to analyse the dynamics of strain gain or loss and variation in gene content over time.

402 Homologous recombination has also been noted to contribute to the generation of diversity

403 (Golubchik et al. 2013; González-González et al. 2013), however, we detected and remove

404 recombinant regions prior to phylogenetic reconstruction and thus focused on our analysis on 405 SNPs. 
More than half of our isolates encode resistance to three or more classes of antimicrobials

407 echoing the high rate of MDR (65\%; confirmed by phenotypic testing) in the GEMS study.

408 IncFIB (AP001918) was the most common plasmid Inc type from our study, in line with the

409 observation that IncF plasmids are frequently associated with the dissemination of resistance

410 (Carattoli 2009). However, a limitation of our study is that we did not perform phenotypic

411 antimicrobial resistance testing, although Doyle et al. (Doyle et al. 2020) reported that only a

412 small proportion of genotypic AMR predictions are discordant with phenotypic results.

413 Comparative analyses confirm the heterogeneous origins of the strains reported here,

414 documenting links to other human commensal strains or isolates sourced from livestock or the

415 environment. This is not surprising, as almost all study participants reported that animals are kept

416 in their homes and children in rural Gambia are often left to play on the ground, close to

417 domestic animals such as pets and poultry (Dione et al. 2011).

418

419 Conclusions

420 Our results show that the commensal E. coli population in the gut of healthy children in rural

421 Gambia is richly diverse, with the independent immigration and establishment of strains

422 contributing to the bulk of the observed diversity. An obvious limitation to our study is the low

423 recovery of E. coli from frozen stools - which potentially implies we may have underestimated

424 the extent of genetic diversity present within our study population. Although solely

425 observational, our study paves the way for future studies aimed at a mechanistic understanding

426 of the factors driving the diversification of E. coli in the human gut and what it takes to make a

427 strain of E. coli successful in this habitat. Besides, this work has added significantly to the

428 number of commensal E. coli genomes, which are underrepresented in public repositories. 
430 Acknowledgements

431 We gratefully acknowledge the study participants in GEMS and all clinicians, field workers and 432 the laboratory staff of the Medical Research Council Unit The Gambia at London School of 433 Hygiene and Tropical Medicine involved in the collection and storage of stools in the GEMS 434 study in Basse Field Station and Fajara.

\section{Author contributions}

437 Conceptualization: MA, MP; data curation, MP, NFA; formal analysis: EFN; analytical support: MD; funding: MA and MP; sample collection and storage: MJH, UNI, PET, CO; data management: SG; laboratory experiments, EFN, supervision, NFA, MP, JO, MA; manuscript preparation - original draft, EFN; review and editing, NFA, MP; review of the final manuscript, 441 all authors.

\section{References}

Ahmed SF, Ali MM, Mohamed ZK, Moussa TA, and Klena JD. 2014. Fecal carriage of extended-spectrum $\beta$-lactamases and AmpC-producing Escherichia coli in a Libyan community. Annals of Clinical Microbiology and Antimicrobials 13:22. 10.1186/14760711-13-22 of Bacteria: American Society of Microbiology

Alm EW, Walk ST, and Gordon DM. 2011. The niche of Escherichia coli. Population Genetics

Bankevich A, Nurk S, Antipov D, Gurevich AA, Dvorkin M, Kulikov AS, Lesin VM, Nikolenko SI, Pham S, Prjibelski AD, Pyshkin AV, Sirotkin AV, Vyahhi N, Tesler G, Alekseyev 
452

453

454

455

456

457

458

459

460

461

462

463

464

465

466

467

468

469

470

471

472

473

474

MA, and Pevzner PA. 2012. SPAdes: a new genome assembly algorithm and its applications to single-cell sequencing. Journal of Computational Biology 19:455-477. $10.1089 / \mathrm{cmb} .2012 .0021$

Bettelheim KA, Faiers M, and Shooter RA. 1972. Serotypes of Escherichia coli in normal stools. The Lancet 2:1223-1224.

Blount ZD. 2015. The unexhausted potential of E. coli. Elife 4. 10.7554/eLife.05826

Camins BC, Marschall J, DeVader SR, Maker DE, Hoffman MW, and Fraser VJ. 2011. The clinical impact of fluoroquinolone resistance in patients with E. coli bacteremia. Journal of Hospital Medicine 6:344-349. 10.1002/jhm.877

Carattoli A. 2009. Resistance plasmid families in Enterobacteriaceae. Antimicrobial Agents and Chemotherapy 53:2227-2238. 10.1128/AAC.01707-08

Chen SL, Wu M, Henderson JP, Hooton TM, Hibbing ME, Hultgren SJ, and Gordon JI. 2013. Genomic diversity and fitness of E. coli strains recovered from the intestinal and urinary tracts of women with recurrent urinary tract infection. Science Translational Medicine 5:184ra160. 10.1126/scitranslmed.3005497

Clermont O, Christenson JK, Denamur E, and Gordon DM. 2013. The Clermont Escherichia coli phylo-typing method revisited: improvement of specificity and detection of new phylogroups. Environmental Microbiology Reports 5:58-65. 10.1111/1758-2229.12019

Clermont O, Gordon D, and Denamur E. 2015. Guide to the various phylogenetic classification schemes for Escherichia coli and the correspondence among schemes. Microbiology 161:980-988. 10.1099/mic.0.000063

Connor TR, Loman NJ, Thompson S, Smith A, Southgate J, Poplawski R, Bull MJ, Richardson E, Ismail M, Thompson SE, Kitchen C, Guest M, Bakke M, Sheppard SK, and Pallen MJ. 
475

476

477

478

479

480

481

482

483

484

485

486

487

488

489

490

491

492

493

494

495

496

2016. CLIMB (the Cloud Infrastructure for Microbial Bioinformatics): an online resource for the medical microbiology community. Microbial Genomics 2:e000086. 10.1099/mgen.0.000086

Croucher NJ, Page AJ, Connor TR, Delaney AJ, Keane JA, Bentley SD, Parkhill J, and Harris SR. 2015. Rapid phylogenetic analysis of large samples of recombinant bacterial whole genome sequences using Gubbins. Nucleic Acids Research 43:e15-e15.

\subsection{3/nar/gku1196}

Dione MM, Ikumapayi UN, Saha D, Mohammed NI, Geerts S, Ieven M, Adegbola RA, and Antonio M. 2011. Clonal differences between Non-Typhoidal Salmonella (NTS) recovered from children and animals living in close contact in the Gambia. PLoS Neglected Tropical Diseases 5:e1148. 10.1371/journal.pntd.0001148

Dixit OVA, O'Brien CL, Pavli P, and Gordon DM. 2018. Within-host evolution versus immigration as a determinant of Escherichia coli diversity in the human gastrointestinal tract. Environmental Microbiology 20:993-1001. 10.1111/1462-2920.14028

Doyle RM, O'Sullivan DM, Aller SD, Bruchmann S, Clark T, Coello Pelegrin A, Cormican M, Diez Benavente E, Ellington MJ, McGrath E, Motro Y, Phuong Thuy Nguyen T, Phelan J, Shaw LP, Stabler RA, van Belkum A, van Dorp L, Woodford N, Moran-Gilad J, Huggett JF, and Harris KA. 2020. Discordant bioinformatic predictions of antimicrobial resistance from whole-genome sequencing data of bacterial isolates: an inter-laboratory study. Microbial Genomics 6. 10.1099/mgen.0.000335

Duriez P, Clermont O, Bonacorsi S, Bingen E, Chaventré A, Elion J, Picard B, and Denamur E. 2001. Commensal Escherichia coli isolates are phylogenetically distributed among 
geographically distinct human populations. Microbiology 147:1671-1676.

499 Escobar-Paramo P, Clermont O, Blanc-Potard AB, Bui H, Le Bouguenec C, and Denamur E. 2004. A specific genetic background is required for acquisition and expression of virulence factors in Escherichia coli. Molecular Biology and Evolution 21:1085-1094.

Escobar-Páramo P, Grenet K, Le Menac'h A, Rode L, Salgado E, Amorin C, Gouriou S, Picard B, Rahimy MC, Andremont A, Denamur E, and Ruimy R. 2004. Large-scale population structure of human commensal Escherichia coli isolates. Applied and Environmental Microbiology 70:5698-5700. 10.1128/AEM.70.9.5698-5700.2004

Ferjani S, Saidani M, Hamzaoui Z, Alonso CA, Torres C, Maamar E, Slim AF, and Boutiba BB. 2017. Community fecal carriage of broad-spectrum cephalosporin-resistant Escherichia coli in Tunisian children. Diagnostic Microbiology and Infectious Disease 87:188-192.

Foster-Nyarko E, Alikhan NF, Ravi A, Thilliez G, Thomson NM, Baker D, Kay G, Cramer JD, O'Grady J, Antonio M, and Pallen MJ. 2020. Genomic diversity of Escherichia coli isolates from non-human primates in the Gambia. Microbial Genomics 6.

Golubchik T, Batty EM, Miller RR, Farr H, Young BC, Larner-Svensson H, Fung R, Godwin H, Knox K, Votintseva A, Everitt RG, Street T, Cule M, Ip CLC, Didelot X, Peto TEA, Harding RM, Wilson DJ, Crook DW, and Bowden R. 2013. Within-host evolution of Staphylococcus aureus during asymptomatic carriage. PLoS One 8:e61319. 10.1371/journal.pone.0061319 
520 González-González A, Sánchez-Reyes LL, Delgado Sapien G, Eguiarte LE, and Souza V. 2013.

521 Hierarchical clustering of genetic diversity associated to different levels of mutation and

522 recombination in Escherichia coli: a study based on Mexican isolates. Infections,

523 Genetics and Evolution 13:187-197. 10.1016/j.meegid.2012.09.003

524 Good BH, McDonald MJ, Barrick JE, Lenski RE, and Desai MM. 2017. The dynamics of molecular evolution over 60,000 generations. Nature 551:45-50. 10.1038/nature24287

526 Gordon DM, O'Brien CL, and Pavli P. 2015. Escherichia coli diversity in the lower intestinal tract of humans. Environmental Microbiology Reports 7:642-648. 10.1111/1758-

528 2229.12300

Gurevich A, Saveliev V, Vyahhi N, and Tesler G. 2013. QUAST: quality assessment tool for genome assemblies. Bioinformatics 29:1072-1075. 10.1093/bioinformatics/btt086

531 Hartl DL, and Dykhuizen DE. 1984. The population genetics of Escherichia coli. Annual Reviews of Genetics 18:31-68. 10.1146/annurev.ge.18.120184.000335

533 Hobman JL, Penn CW, and Pallen MJ. 2007. Laboratory strains of Escherichia coli: model citizens or deceitful delinquents growing old disgracefully? Molecular Microbiology 64:881-885. 10.1111/j.1365-2958.2007.05710.x

Kotloff KL, Blackwelder WC, Nasrin D, Nataro JP, Farag TH, van Eijk A, Adegbola RA, Alonso PL, Breiman RF, Faruque AS, Saha D, Sow SO, Sur D, Zaidi AK, Biswas K, Panchalingam S, Clemens JD, Cohen D, Glass RI, Mintz ED, Sommerfelt H, and Levine MM. 2012. The Global Enteric Multicenter Study (GEMS) of diarrheal disease in infants and young children in developing countries: epidemiologic and clinical methods of the case/control study. Clinical Infectious Diseases 55 Suppl 4:S232-245. 10.1093/cid/cis753 
542 Kotloff KL, Nataro JP, Blackwelder WC, Nasrin D, Farag TH, Panchalingam S, Wu Y, Sow SO,

543 Sur D, Breiman RF, Faruque ASG, Zaidi AKM, Saha D, Alonso PL, Tamboura B,

544 Sanogo D, Onwuchekwa U, Manna B, Ramamurthy T, Kanungo S, Ochieng JB, Omore

545 R, Oundo JO, Hossain A, Das SK, Ahmed S, Qureshi S, Quadri F, Adegbola RA,

546 Antonio M, Hossain MJ, Akinsola A, Mandomando I, Nhampossa T, Acácio S, Biswas

547 K, O'Reilly CE, Mintz ED, Berkeley LY, Muhsen K, Sommerfelt H, Robins-Browne

548 RM, and Levine MM. 2013. Burden and aetiology of diarrhoeal disease in infants and

549 young children in developing countries (the Global Enteric Multicenter Study, GEMS): a

$550 \quad$ prospective, case-control study. The Lancet 382:209-222. 10.1016/S0140-

551

6736(13)60844-2

552 Laxminarayan R, Duse A, Wattal C, Zaidi AK, Wertheim HF, Sumpradit N, Vlieghe E, Hara

GL, Gould IM, Goossens H, Greko C, So AD, Bigdeli M, Tomson G, Woodhouse W,

Ombaka E, Peralta AQ, Qamar FN, Mir F, Kariuki S, Bhutta ZA, Coates A, Bergstrom R,

555 Wright GD, Brown ED, and Cars O. 2013. Antibiotic resistance-the need for global

556 solutions. The Lancet Infectious Diseases 13:1057-1098. 10.1016/s1473-3099(13)70318-

557 9

Li H, Handsaker B, Wysoker A, Fennell T, Ruan J, Homer N, Marth G, Abecasis G, Durbin R, and Subgroup GPDP. 2009. The Sequence Alignment/Map format and SAMtools. Bioinformatics 25:2078-2079. 10.1093/bioinformatics/btp352

Lidin-Janson G, Kaijser B, Lincoln K, Olling S, and Wedel H. 1978. The homogeneity of the 562 faecal coliform flora of normal school-girls, characterized by serological and biochemical properties. Medical Microbiology and Immunology 164:247-253. 10.1007/BF02125493 
564 Liu J, Platts-Mills JA, Juma J, Kabir F, Nkeze J, Okoi C, Operario DJ, Uddin J, Ahmed S,

565 Alonso PL, Antonio M, Becker SM, Blackwelder WC, Breiman RF, Faruque AS, Fields

566 B, Gratz J, Haque R, Hossain A, Hossain MJ, Jarju S, Qamar F, Iqbal NT, Kwambana B,

567 Mandomando I, McMurry TL, Ochieng C, Ochieng JB, Ochieng M, Onyango C,

568 Panchalingam S, Kalam A, Aziz F, Qureshi S, Ramamurthy T, Roberts JH, Saha D, Sow

569 SO, Stroup SE, Sur D, Tamboura B, Taniuchi M, Tennant SM, Toema D, Wu Y, Zaidi A,

$570 \quad$ Nataro JP, Kotloff KL, Levine MM, and Houpt ER. 2016. Use of quantitative molecular

571 diagnostic methods to identify causes of diarrhoea in children: a reanalysis of the GEMS

572 case-control study. Lancet 388:1291-1301. 10.1016/S0140-6736(16)31529-X

573 Massot M, Daubié AS, Clermont O, Jauréguy F, Couffignal C, Dahbi G, Mora A, Blanco J,

574 Branger C, Mentré F, Eddi A, Picard B, Denamur E, and The Coliville Group. 2016.

575 Phylogenetic, virulence and antibiotic resistance characteristics of commensal strain

576 populations of Escherichia coli from community subjects in the Paris area in 2010 and

577 evolution over 30 years. Microbiology 162:642-650. 10.1099/mic.0.000242

578 McNally A, Alhashash F, Collins M, Alqasim A, Paszckiewicz K, Weston V, and Diggle M.

579 2013. Genomic analysis of extra-intestinal pathogenic Escherichia coli urosepsis.

Clinical Microbiology and Infection 19:E328-334. 10.1111/1469-0691.12202

581 Mellata M. 2013. Human and avian extraintestinal pathogenic Escherichia coli: infections, zoonotic risks, and antibiotic resistance trends. Foodborne Pathogens and Disease 10:916-932. 10.1089/fpd.2013.1533

Milne I, Stephen G, Bayer M, Cock PJ, Pritchard L, Cardle L, Shaw PD, and Marshall D. 2013. Using Tablet for visual exploration of second-generation sequencing data. Briefings in Bioinformatics 14:193-202. 10.1093/bib/bbs012 
587 Moremi N, Claus H, Vogel U, and Mshana SE. 2017. Faecal carriage of CTX-M extended-

588 spectrum beta-lactamase-producing Enterobacteriaceae among street children dwelling

589 in Mwanza city, Tanzania. PLoS One 12:e0184592. 10.1371/journal.pone.0184592

590 Nielsen KL, Stegger M, Godfrey PA, Feldgarden M, Andersen PS, and Frimodt-Møller N. 2016.

591 Adaptation of Escherichia coli traversing from the faecal environment to the urinary

592 tract. International Journal of Medical Microbiology 306:595-603.

$593 \quad 10.1016 /$ j.ijmm.2016.10.005

594 Oshima K, Toh H, Ogura Y, Sasamoto H, Morita H, Park SH, Ooka T, Iyoda S, Taylor TD,

595 Hayashi T, Itoh K, and Hattori M. 2008. Complete genome sequence and comparative

596 analysis of the wild-type commensal Escherichia coli strain SE11 isolated from a healthy

597 adult. DNA Research 15:375-386. 10.1093/dnares/dsn026

598 Pokharel P, Habouria H, Bessaiah H, and Dozois CM. 2019. Serine Protease Autotransporters of 599 the Enterobacteriaceae (SPATEs): Out and about and chopping it up. Microorganisms 7.

$600 \quad 10.3390 /$ microorganisms 7120594

601 Rasko DA, Rosovitz MJ, Myers GS, Mongodin EF, Fricke WF, Gajer P, Crabtree J, Sebaihia M, 602 Thomson NR, Chaudhuri R, Henderson IR, Sperandio V, and Ravel J. 2008. The 603 604 605 pangenome structure of Escherichia coli: comparative genomic analysis of E. coli 2011. Rates of mutation and host transmission for an Escherichia coli clone over 3 years. 608 PloS One 6:e26907-e26907. 10.1371/journal.pone.0026907 
609 Richter TKS, Hazen TH, Lam D, Coles CL, Seidman JC, You Y, Silbergeld EK, Fraser CM, and

610 Rasko DA. 2018. Temporal variability of Escherichia coli diversity in the gastrointestinal

611 tracts of Tanzanian children with and without exposure to antibiotics. mSphere 3.

$612 \quad 10.1128 / \mathrm{mSphere} .00558-18$

613 Rodríguez-Baño J, Picón E, Gijón P, Hernández JR, Cisneros JM, Peña C, Almela M, Almirante

B, Grill F, Colomina J, Molinos S, Oliver A, Fernández-Mazarrasa C, Navarro G,

615 Coloma A, López-Cerero L, and Pascual A. 2010. Risk factors and prognosis of nosocomial bloodstream infections caused by extended-spectrum-beta-lactamase-

618 producing Escherichia coli. Journal of Clinical Microbiology 48:1726-1731.

Russo TA, and Johnson JR. 2003. Medical and economic impact of extraintestinal infections due to Escherichia coli: focus on an increasingly important endemic problem. Microbes and Infection 5:449-456. 10.1016/s1286-4579(03)00049-2

Schlager TA, Hendley JO, Bell AL, and Whittam TS. 2002. Clonal diversity of Escherichia coli colonizing stools and urinary tracts of young girls. Infection and Immunity 70:1225-1229.

Shooter RA, Bettleheim KA, Lennox-King SM, and O'Farrell S. 1977. Escherichia coli 10.1128/iai.70.3.1225-1229.2002 serotypes in the faeces of healthy adults over a period of several months. Journal of Hygiene (Lond) 78:95-98. 10.1017/s0022172400055972

Skurnik D, Bonnet D, Bernède-Bauduin C, Michel R, Guette C, Becker JM, Balaire C, Chau F, Mohler J, Jarlier V, Boutin JP, Moreau B, Guillemot D, Denamur E, Andremont A, and Ruimy R. 2008. Characteristics of human intestinal Escherichia coli with changing 
633 Stamatakis A. 2006. RAxML-VI-HPC: maximum likelihood-based phylogenetic analyses with thousands of taxa and mixed models. Bioinformatics 22:2688-2690.

Stegger M, Leihof RF, Baig S, Sieber RN, Thingholm KR, Marvig RL, Frimodt-Møller N, and Nielsen KL. 2020. A snapshot of diversity: Intraclonal variation of Escherichia coli clones as commensals and pathogens. International Journal of Medical Microbiology 310:151401. 10.1016/j.ijmm.2020.151401

Stoesser N, Sheppard AE, Moore CE, Golubchik T, Parry CM, Nget P, Saroeun M, Day NP, Giess A, Johnson JR, Peto TE, Crook DW, Walker AS, and Group MMMI. 2015. Extensive within-host diversity in fecally carried extended-spectrum-beta-lactamaseproducing Escherichia coli isolates: Implications for transmission analyses. Journal of

Tenaillon O, Skurnik D, Picard B, and Denamur E. 2010. The population genetics of commensal Escherichia coli. Nature Reviews Microbiology 8:207-217. 10.1038/nrmicro2298 Bouchier C, Bouvet O, Calteau A, Chiapello H, Clermont O, Cruveiller S, Danchin A, Diard M, Dossat C, Karoui ME, Frapy E, Garry L, Ghigo JM, Gilles AM, Johnson J, Le 
654

655

656

657

658

659

660

661

662

663

664

665

666

667

668

669

670

671

672

673

674

675

676

Bouguénec C, Lescat M, Mangenot S, Martinez-Jéhanne V, Matic I, Nassif X, Oztas S, Petit MA, Pichon C, Rouy Z, Ruf CS, Schneider D, Tourret J, Vacherie B, Vallenet D, Médigue C, Rocha EP, and Denamur E. 2009. Organised genome dynamics in the Escherichia coli species results in highly diverse adaptive paths. PLoS Genetics 5:e1000344. 10.1371/journal.pgen.1000344

Walk ST, Alm EW, Gordon DM, Ram JL, Toranzos GA, Tiedje JM, and Whittam TS. 2009. Cryptic lineages of the genus Escherichia. Applied and Environmental Microbioogy 75:6534-6544. 10.1128/aem.01262-09

Wheeler TJ. 2009. Large-Scale Neighbor-Joining with NINJA. in Algorithms in Bioinformatics. Berlin, Heidelberg: Springer Berlin Heidelberg.

Wingett SW, and Andrews S. 2018. FastQ Screen: A tool for multi-genome mapping and quality control. F1000Res 7:1338. 10.12688/f1000research.15931.2

Wirth T, Falush D, Lan R, Colles F, Mensa P, Wieler LH, Karch H, Reeves PR, Maiden MCJ, Ochman H, and Achtman M. 2006. Sex and virulence in Escherichia coli: an evolutionary perspective. Molecular Microbiology 60:1136-1151. 10.1111/j.13652958.2006.05172.x

Wold AE, Caugant DA, Lidin-Janson G, de Man P, and Svanborg C. 1992. Resident colonic Escherichia coli strains frequently display uropathogenic characteristics. Journal of Infectious Diseases 165:46-52. 10.1093/infdis/165.1.46

Zhou Z, Alikhan NF, Mohamed K, Fan Y, Achtman M, and Group AS. 2020. The EnteroBase user's guide, with case studies on Salmonella transmissions, Yersinia pestis phylogeny, and Escherichia core genomic diversity. Genome Research 30:138-152.

$10.1101 /$ gr.251678.119

Peer) reviewing PDF | (2020:09:52546:1:1:NEW 2 Nov 2020) 


\section{Table $\mathbf{1}$ (on next page)}

Phylogroup and sequence types of the distinct clones isolated in each study subject 
2 Table 1: Phylogroup and sequence types of the distinct clones isolated in each study subject

\begin{tabular}{|c|c|c|c|c|c|c|c|c|}
\hline \multicolumn{6}{|c|}{ Colony or isolate number } & \multirow{2}{*}{$\begin{array}{c}\text { Number of } \\
\text { distinct } \\
\text { genotypes } \\
\text { (clones) }\end{array}$} & \multicolumn{2}{|r|}{ Within-host evolution events } \\
\hline Host & 1 & 2 & 3 & 4 & 5 & & Phylotype (number of events) & Phylotype (number of events) \\
\hline$H-2$ & A (9274) & A (9274) & A (9274) & A (9274) & A (9274) & 1 & $\mathrm{~A}(1)$ & 0 \\
\hline$H-9$ & A (2705) & A (2705) & A (2705) & $\mathrm{D}(2914)$ & B1 (29) & 3 & $\mathrm{~A}(1), \mathrm{D}(1), \mathrm{B} 1(1)$ & 0 \\
\hline$H-15$ & $\begin{array}{l}\text { B2 } \\
(9277)\end{array}$ & $\begin{array}{l}\text { B2 } \\
(9277)\end{array}$ & $\begin{array}{l}\text { B2 } \\
\text { (9277) }\end{array}$ & $\begin{array}{l}\text { Clade I } \\
\text { (747) }\end{array}$ & $\begin{array}{l}\text { Clade I } \\
\text { (747) }\end{array}$ & 3 & B2 (1), Clade I (1) & Clade I (1) \\
\hline$H-18$ & $\mathrm{D}(38)$ & $\mathrm{D}(38)$ & $\begin{array}{l}\text { B1 } \\
(9281)\end{array}$ & A (9274) & & 4 & $\mathrm{D}(1), \mathrm{B} 1(1), \mathrm{A}(1)$ & $\mathrm{D}(1)$ \\
\hline$H-21$ & B1 (58) & B1 (58) & B1 (223) & A (540) & $\mathrm{D}(1204)$ & 4 & B1(2) A (1), D (1) & 0 \\
\hline$H-22$ & B1 (316) & B1 (316) & B1 (316) & B1 (316) & & 2 & $\mathrm{~B}(1)$ & B1(1) \\
\hline$H-25$ & A (181) & $\mathrm{A}(181)$ & A (181) & $\mathrm{A}(181)$ & B1 (337) & 4 & A (1), B1 (1) & $\mathrm{A}(2)$ \\
\hline$H-26$ & B1 (641) & $\begin{array}{l}\text { B1 } \\
\text { (2741) }\end{array}$ & $\mathrm{A}(10)$ & A (398) & & 4 & $\mathrm{~B} 1(2), \mathrm{A}(1), \mathrm{D}(1)$ & 0 \\
\hline$H-28$ & B1 (469) & B1 (469) & B1 (469) & B1 (469) & & 2 & $\mathrm{~B} 1(1)$ & $\mathrm{B} 1(1)$ \\
\hline$H-32$ & B1 (101) & B1 (101) & B1 (101) & $\begin{array}{l}\text { B1 } \\
\text { (2175) }\end{array}$ & $\mathrm{A}(10)$ & 3 & $\mathrm{~B} 1(2), \mathrm{A}(1)$ & 0 \\
\hline$H-34$ & B1 (603) & B1 (603) & B1 (603) & $\begin{array}{l}\text { B1 } \\
\text { (1727) }\end{array}$ & $\mathrm{A}(10)$ & 4 & B1(2), A (1) & $\mathrm{B} 1(1)$ \\
\hline$H-35$ & A (226) & & & & & 1 & A (1) & 0 \\
\hline$H-36$ & $F(59)$ & $F(59)$ & $F(59)$ & $F(59)$ & E (9278) & 4 & $\mathrm{~F}(1), \mathrm{E}(1)$ & $\mathrm{F}(1)$ \\
\hline$H-37$ & $\mathrm{D}(5148)$ & $\mathrm{D}(5148)$ & $\mathrm{D}(5148)$ & $\mathrm{D}(5148)$ & $\mathrm{D}(5148)$ & 3 & $\mathrm{D}(1)$ & $\mathrm{D}(2)$ \\
\hline$H-38$ & D (394) & D (394) & D (394) & D (394) & B1 (58) & 4 & $\mathrm{D}(1), \mathrm{B} 1(1)$ & $\mathrm{D}(2)$ \\
\hline$H-39$ & B2 (452) & B2 (452) & B2 (452) & B2 (452) & B2 (452) & 2 & $\mathrm{~B} 2(1)$ & B2 (1) \\
\hline$H-40$ & B1 (155) & & & & & 1 & B1(1) & 0 \\
\hline$H-41$ & A (43) & A (43) & A (43) & A (43) & $\begin{array}{l}\text { B1 } \\
\text { (9283) }\end{array}$ & 2 & $\mathrm{~A}(1), \mathrm{B} 1(1)$ & 0 \\
\hline$H-48$ & $\begin{array}{l}\text { Clade I } \\
(485)\end{array}$ & $\begin{array}{l}\text { Clade I } \\
(485)\end{array}$ & $\begin{array}{l}\text { Clade I } \\
(485)\end{array}$ & $\begin{array}{l}\text { Clade I } \\
(485)\end{array}$ & & 3 & Clade I (1) & 0 \\
\hline$H-50$ & $\mathrm{C}(410)$ & C (410) & C (410) & C (410) & B1 (515) & 2 & $\mathrm{C}(1), \mathrm{B} 1(1)$ & 0 \\
\hline$H-55$ & A (9279) & & & & & 1 & $\mathrm{~A}(1)$ & 0 \\
\hline
\end{tabular}

3 
Table 2 (on next page)

Pairwise SNP distances between variants arising from within-host evolution 
1 Table 2: Pairwise SNP distances between variants arising from within-host evolution

\begin{tabular}{|c|c|c|c|}
\hline Host & Sequence type (ST) & Colonies per ST & $\begin{array}{c}\text { Pairwise SNP distances between multiple } \\
\text { colonies of the same ST }\end{array}$ \\
\hline$H 2$ & 9274 & 5 & $0-9$ \\
\hline H9 & 2705 & 3 & $0-1$ \\
\hline H15 & 9277 & 3 & $0-1$ \\
\hline H15 & 747 & 2 & 3 \\
\hline H18 & 38 & 2 & 3 \\
\hline H21 & 58 & 2 & 0 \\
\hline H22 & 316 & 4 & $0-3$ \\
\hline H25 & 181 & 4 & $1-5$ \\
\hline H28 & 469 & 4 & $0-3$ \\
\hline H32 & 101 & 3 & $1-9$ \\
\hline H34 & 603 & 3 & $2-8$ \\
\hline H36 & 59 & 4 & $0-14$ \\
\hline H37 & 5148 & 5 & $2-18$ \\
\hline $\mathrm{H38}$ & 394 & 4 & $1-3$ \\
\hline H39 & 452 & 5 & $0-2$ \\
\hline H41 & 43 & 4 & $0-1$ \\
\hline $\mathrm{H} 48$ & 485 & 4 & $1-9$ \\
\hline H50 & 410 & 4 & 0 \\
\hline
\end{tabular}

2 
Figure 1

\section{The study sample-processing flow diagram.}

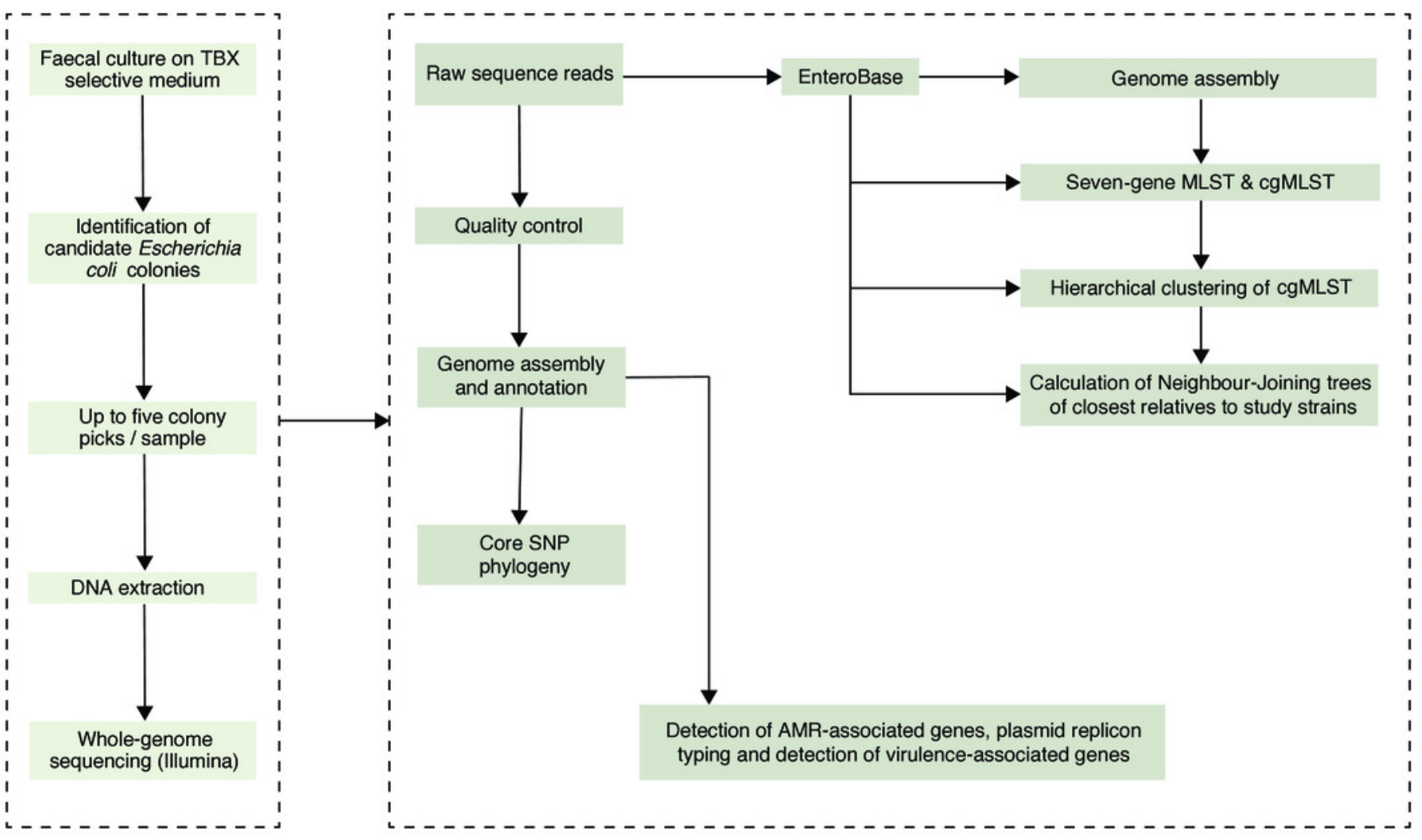




\section{Figure 2}

A maximum-likelihood tree depicting the phylogenetic relationships among the study isolates.

The tree was reconstructed with RAXML, using a general time-reversible nucleotide substitution model and 1,000 bootstrap replicates. The genome assembly of $E$. coli str. K12 substr. MG1655 was used s as the reference, and the tree rooted using the genomic assembly of $E$. fergusonii as an outgroup. The sample names are indicated at the tip, with the respective Achtman sequence types (ST) indicated beside the sample names. The respective phylogroups the isolates belong to are indicated with colour codes as displayed in the legend. E. coli reference genome is denoted in black. Asterisks $(*)$ are used to indicate novel STs. The predicted antimicrobial resistance genes and putative virulence factors for each isolate are displayed next to the tree, with the virulence genes clustered according to their function. Multiple copies of the same strain (ST) isolated from a single host are not shown. Instead, we have shown only one representative isolate from each strain. Virulence and resistance factors were not detected in the reference strain either. A summary of the identified virulence factors and their known functions are provided in Supplementary File 3. 


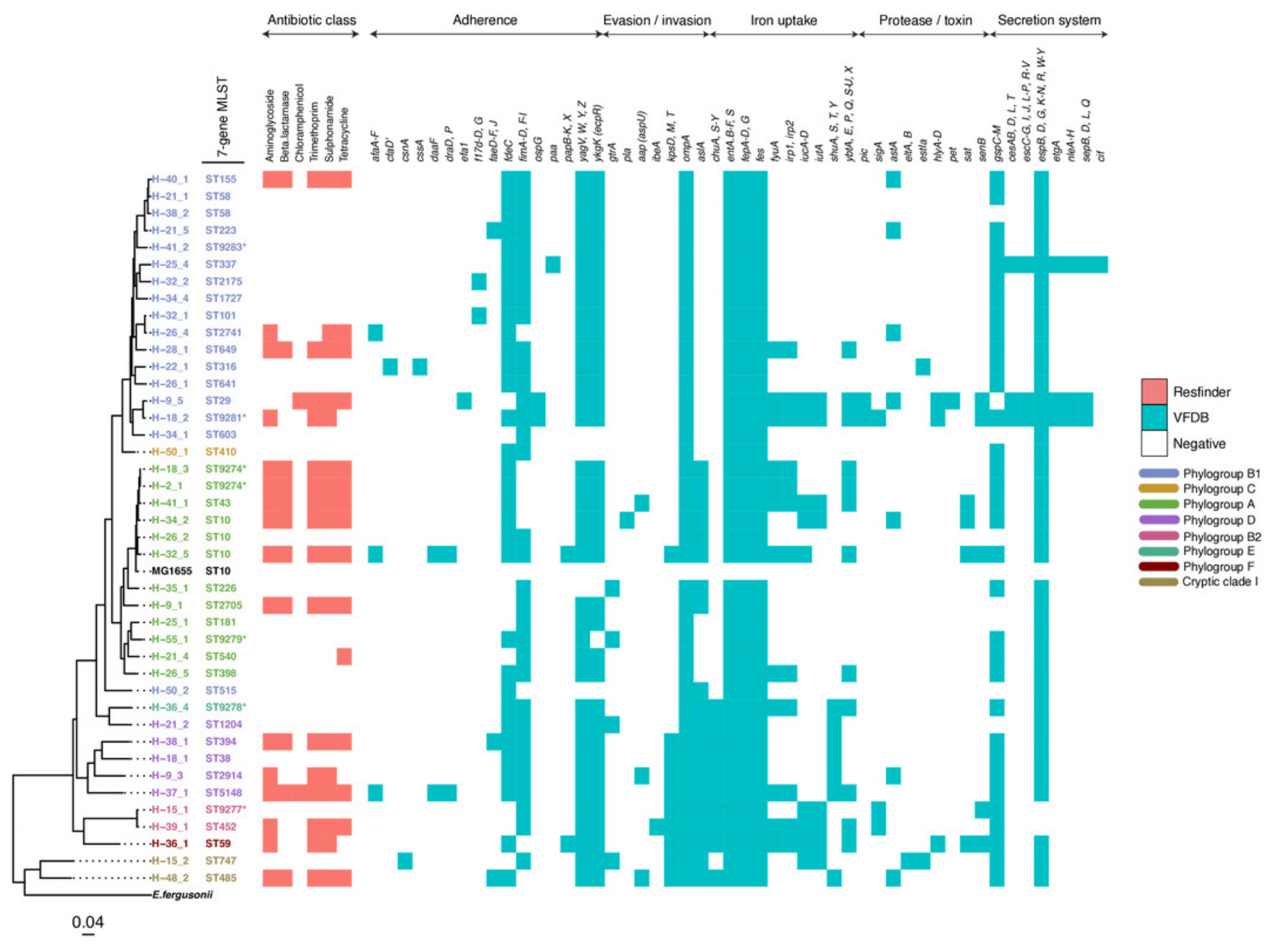




\section{Figure 3}

The population structure of ST38.

A: A NINJA neighbour-joining tree showing the population structure of $E$. coli ST38, drawn using the genomes found in the core-genome MLST hierarchical cluster at HC1100, which corresponds to ST38 clonal complex. The size of the nodes represents the number of isolates per clade. The geographical locations where isolates were recovered are displayed in the legend; with the genome counts shown in square brackets. The study resident ST38 strains and the pathogenic ST38 strains recovered from GEMS cases are highlighted with red circles around the nodes. B: The closest neighbour to a pathogenic strain reported in GEMS [reference 30] is shown to be a commensal isolate recovered from a healthy individual. The size of the nodes represents the number of isolates per clade. The geographical locations where isolates were recovered are displayed in the legend; with the genome counts shown in square brackets. Red circles around the nodes are used to highlight the study resident ST38 strains and the pathogenic ST38 strains recovered from GEMS cases within this cluster. C: The closest relatives to the commensal ST38 strain recovered from this study is shown (red highlights), with the number of core-genome MLST alleles separating the two genomes displayed. The geographical locations where isolates were recovered are displayed in the legend; with the genome counts shown in square brackets, with the size of the nodes depicting the number of isolates per clade. D: A maximum-likelihood phylogenetic tree reconstructed using the genomes found in the cluster in $\mathrm{C}$ above, comprising both pathogenic and commensal ST38 strains is presented, depicting the genetic relationship between strain 100415 (pathogenic) and 103709 (commensal) (red highlights). The nodes are coloured to depict the status of the strains as pathogenic (red) or commensal (blue). The size of the nodes represents the number of isolates per clade. The geographical locations where isolates were recovered are displayed in the legend; with the genome counts shown in 


\section{square brackets.}

A
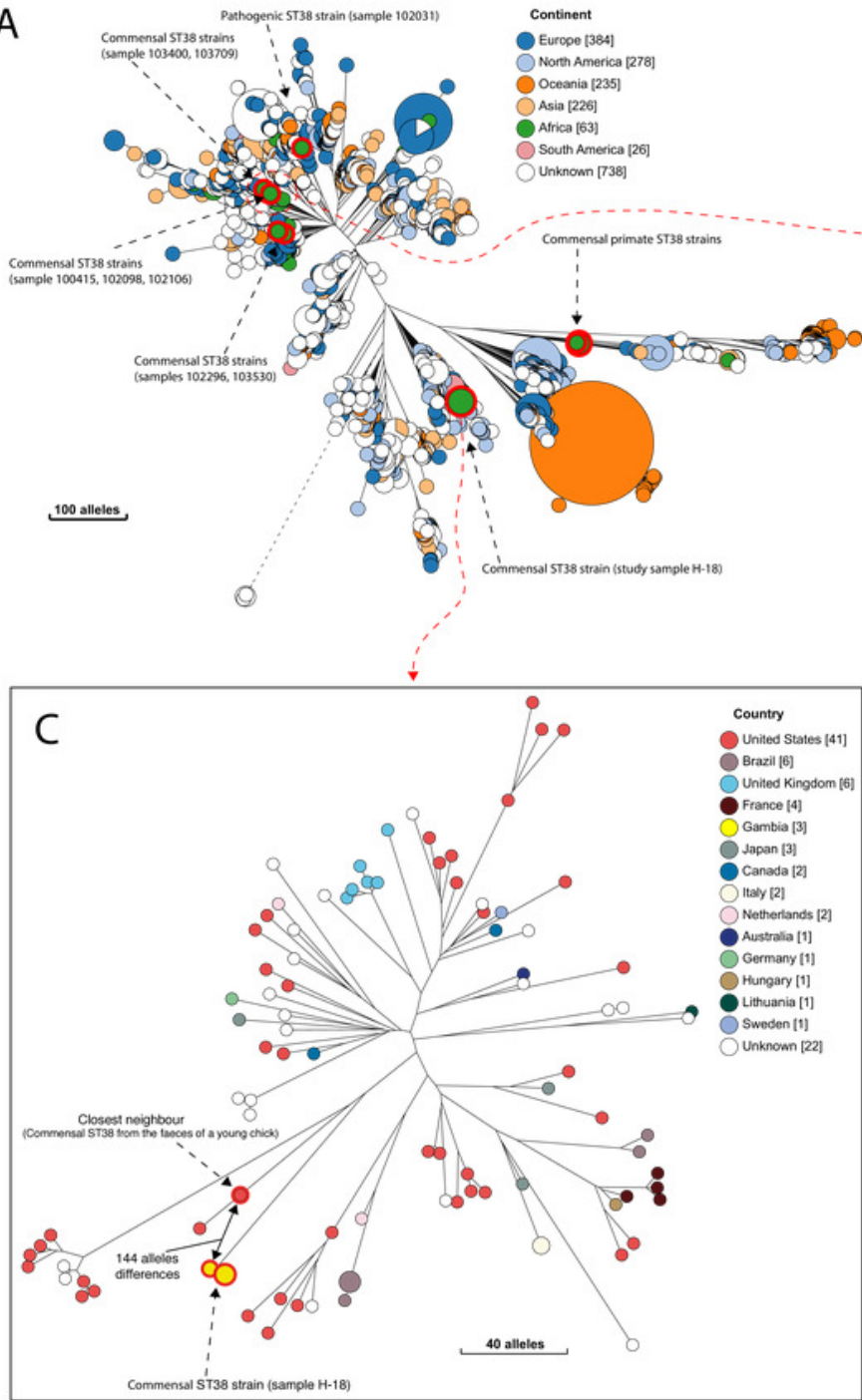

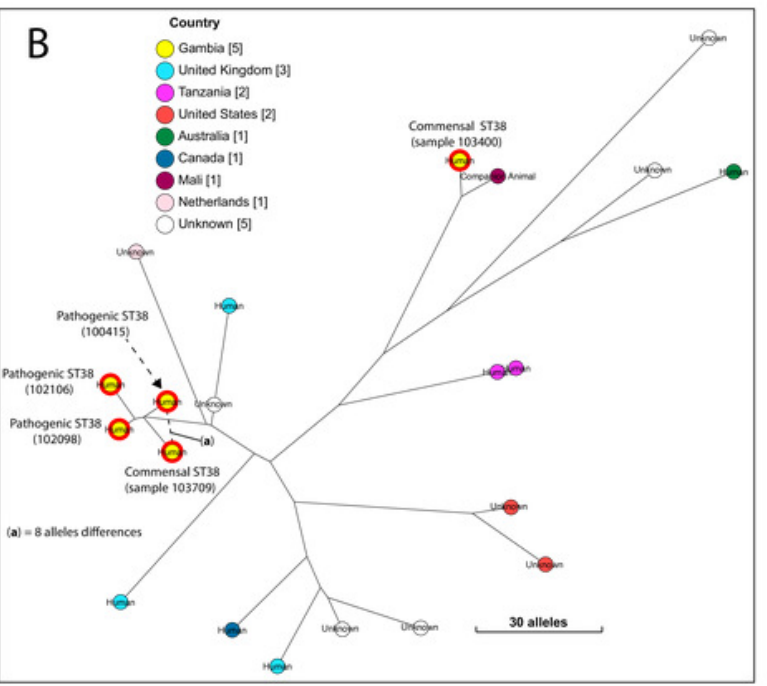

$\mathrm{D}$

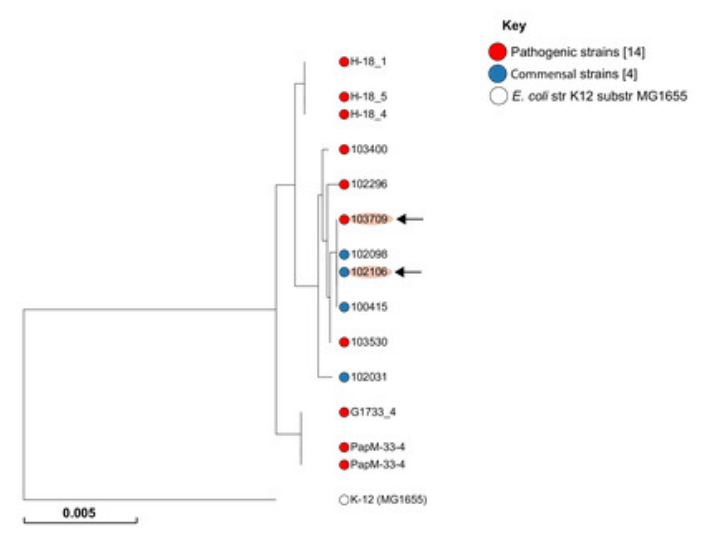




\section{Figure 4}

The population structure of ST58.

A: A NINJA neighbour-joining tree depicting the population structure of E. coli ST58, drawn using the genomes found that clustered together in the same in $\mathrm{HC} 1100$ hierarchical cluster in the core-genome MLST scheme. Commensal ST58 strains from this study and Gambian pathogenic ST58 isolates from GEMS are highlighted in red. The geographical locations where isolates were recovered are displayed in the legend; with the genome counts shown in square brackets. The size of the nodes represents the number of isolates per clade. B and C: The closest relatives to the study ST58 strains are shown. Geographical locations where isolates were recovered are displayed in the legend, with the genome counts displayed in square brackets. The red highlights around the nodes depict the study commensal ST58 strains and their closest neighbours. The size of the nodes represents the number of isolates per clade, and the geographical locations where isolates were recovered are displayed in the legend; with the genome counts shown in square brackets. 


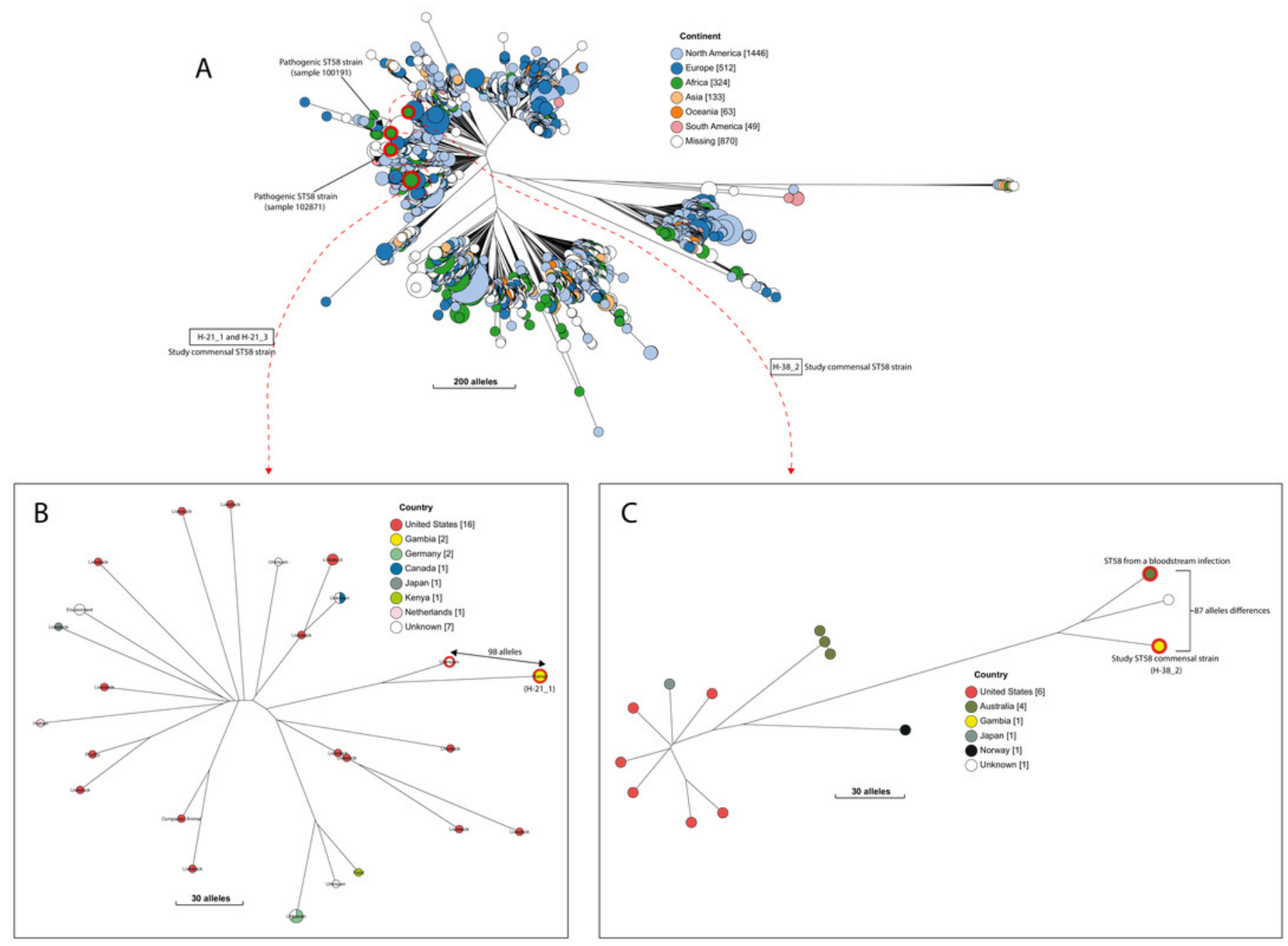




\section{Figure 5}

The prevalence of antimicrobial-associated genes detected in the isolates.

(A) The $y$-axis shows the prevalence of the detected AMR-associated genes in the study isolates, grouped by antimicrobial class. (B) A histogram depicting the number of antimicrobial classes to which resistance genes were detected in the corresponding strains. 
A

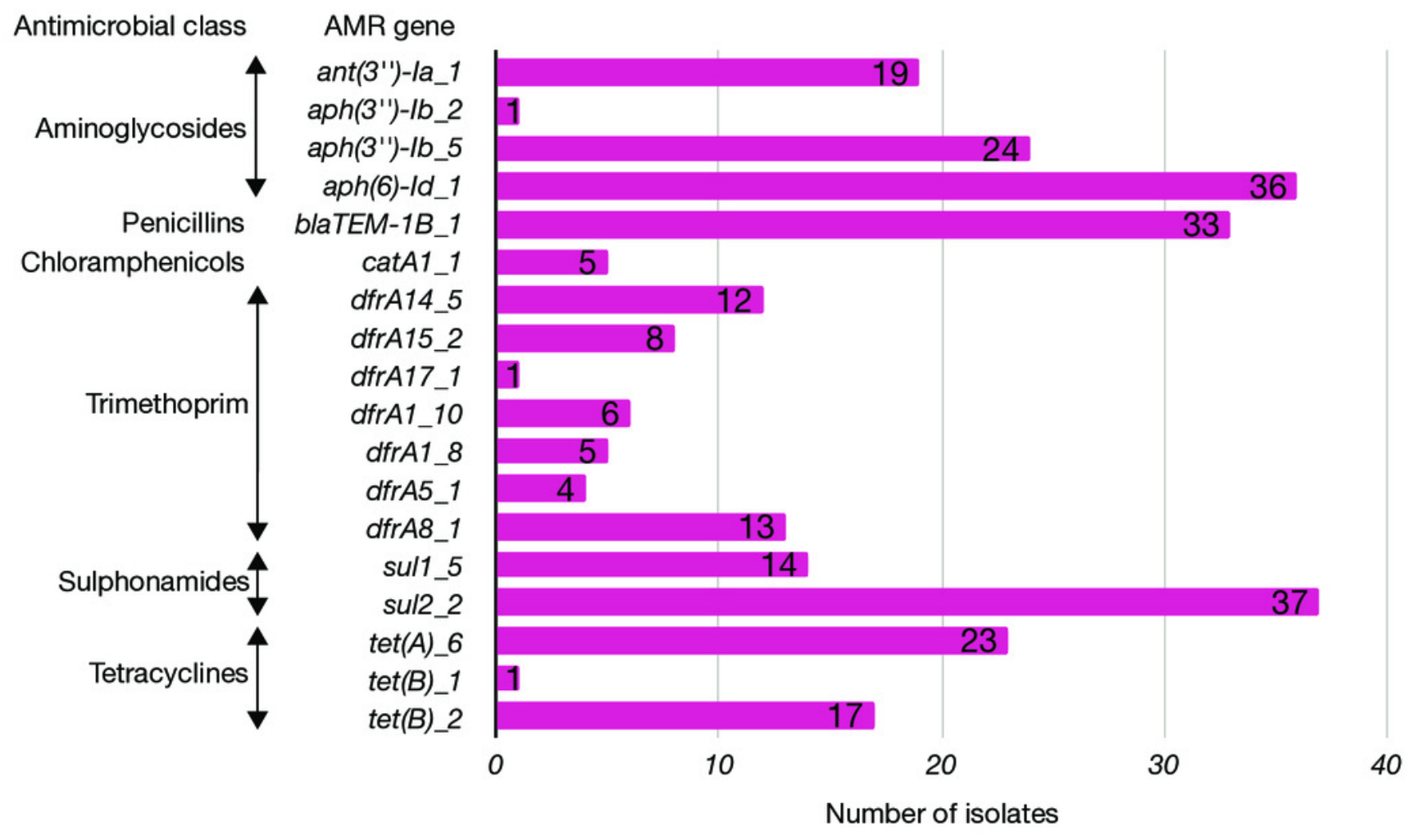

B

30

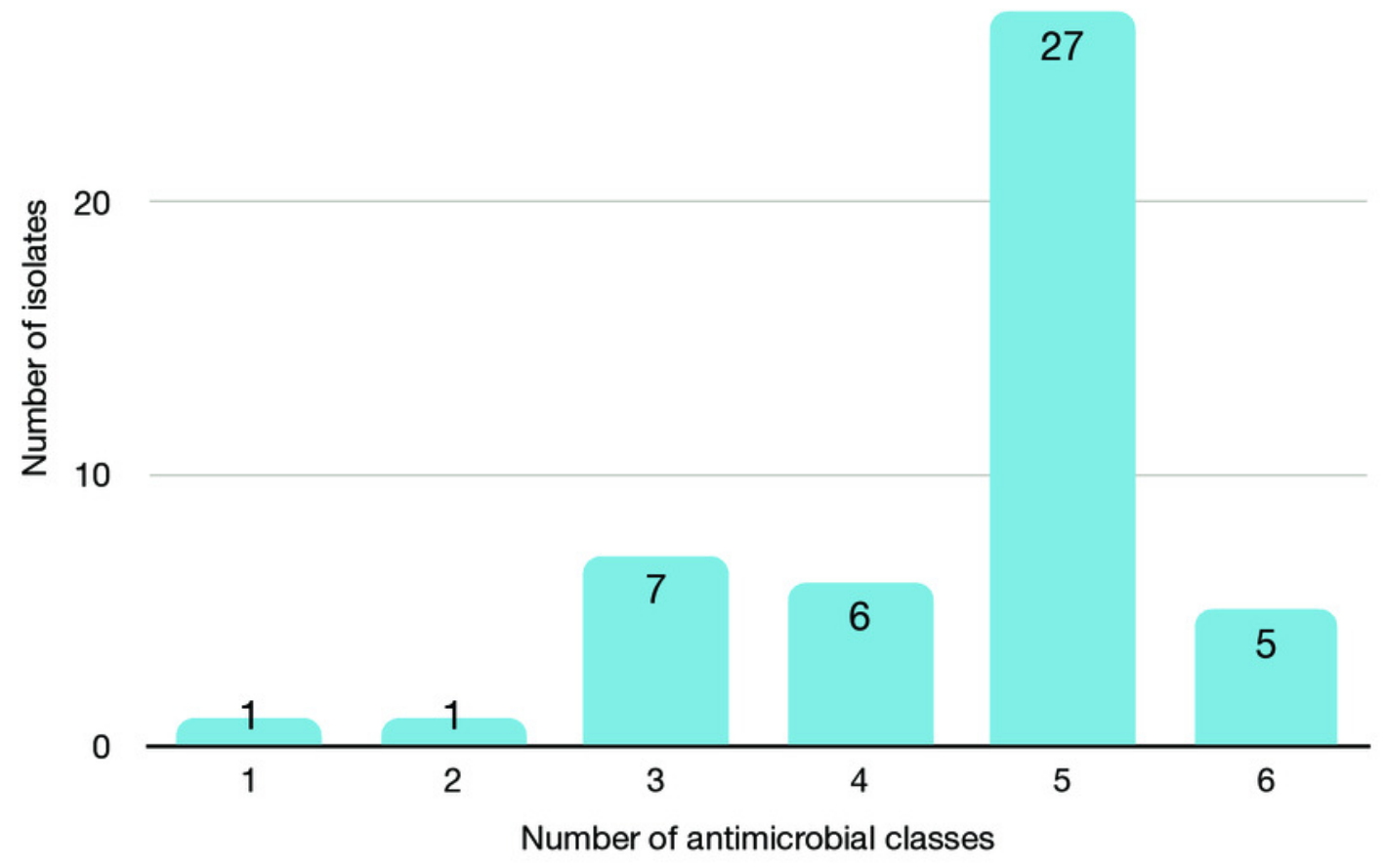


Figure 6

Prevalence of plasmid replicons among the study isolates.

(A) Plasmid replicons detected in the study isolates. (B) A histogram depicting the number of plasmids co-harboured in a single strain. 


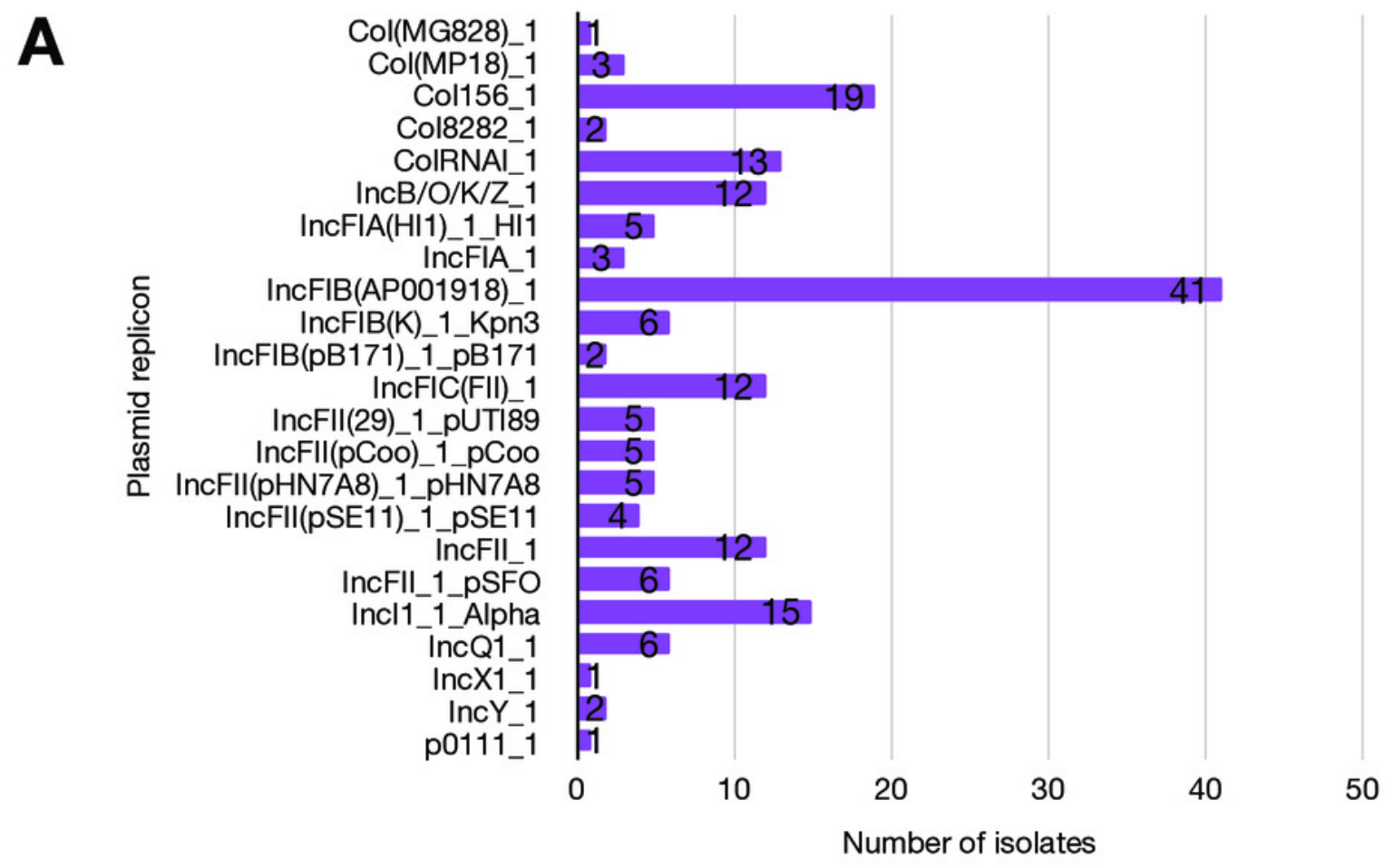

B 30

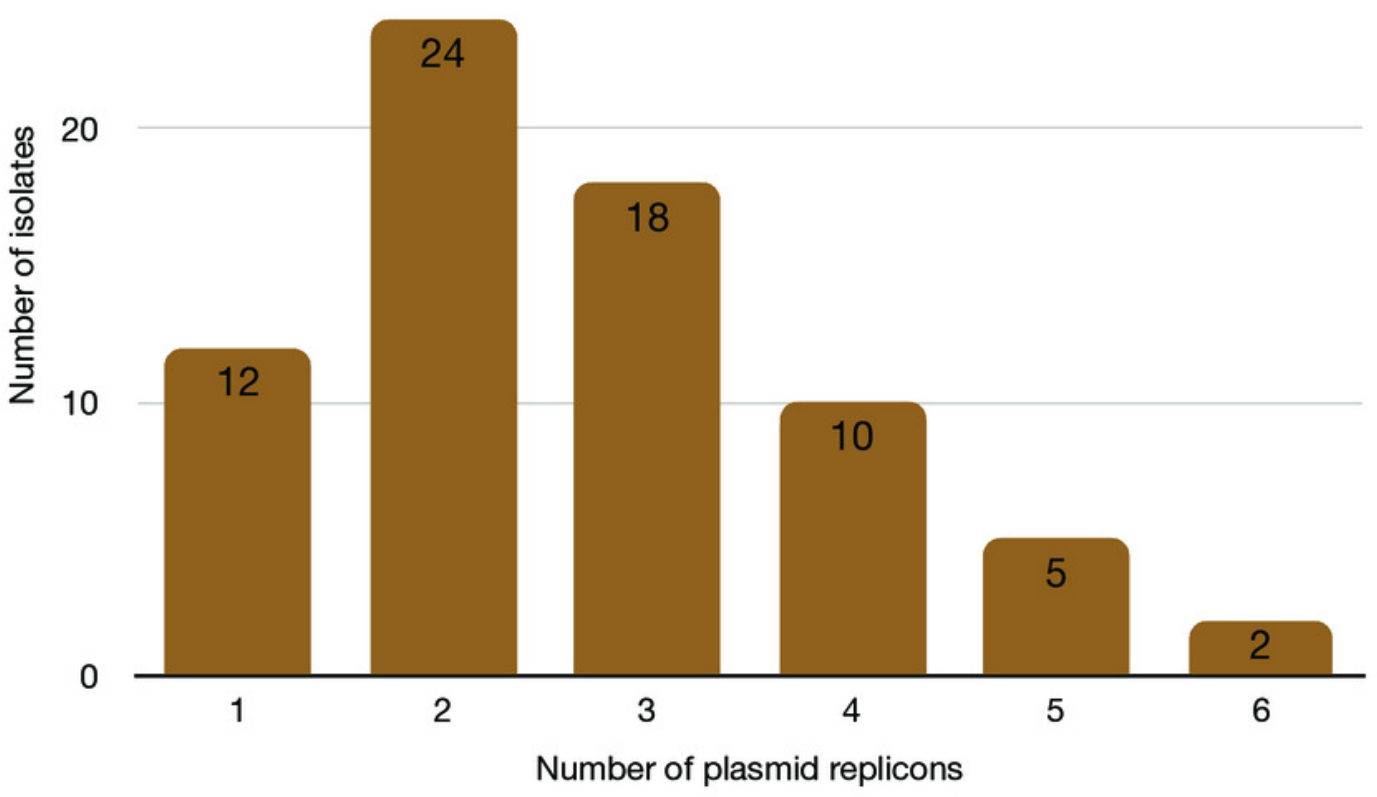

